\title{
13
}

\section{Fault-tolerant UIO Sequences in Finite State Machines}

\author{
Kshirasagar Naik
}

Computer Networks Laboratory, Department of Computer Software, University of Aizu, Aizu-Wakamatsu City, Fukushima, 965-80 JAPAN

\begin{abstract}
The central idea in the paper is to define the concept of strength of a UIO sequence in verifying a state in a faulty implementation. The strength of a UIO sequence is a quantitative measure of the number of output faults required to render the verification power of the sequence ineffective. Thus, UIO sequences of higher strengths can tolerate more output faults while verifying a state. We present an algorithm to compute all UIO sequences of maximal strength in a state machine.
\end{abstract}

Key Words: FSM, Protocol Testing, State Verification, UIO Sequence, Fault Detection, Strength of a UIO Sequence, Fault-tolerant UIO Sequence 


\section{Introduction}

Testing of communication protocols remains to be a subject of continued research. The four test sequence generation methods are the transition tour, the D-, W-, and U-method [SL 89]. The last three are called formal methods, because they verify the next state of a transition. State verification is the main argument in favor of using formal methods over the transition tour method. However, a verification sequence fails to verify the next state of a transition if the verification sequence loses its strength to verify the state due to faults in the implementation. By losing strength, we mean the verification sequence in the specification is not a verification sequence in the implementation. Chan, et. al. showed how a UIO sequence in a specification no more remains a UIO sequence in a faulty implementation [CVI 89].

Once a UIO sequence loses its property to verify a state, there is the danger of faults being undetected. However, in a faulty implementation, there is every likelihood that some UIO sequences lose their property. Given an FSM, UIO sequences can be considered as static properties of the FSM. Or, we can say that the verification power of UIO sequences are static properties of the FSM. In our opinion, on the other hand, faults in an implementation are dynamic "properties" of the implementation. These are dynamic in the sense that one specification FSM can have multiple faulty implementations with one implementation being different from another. While using UIO sequences for state verification, we are faced with the problem of coping with dynamic properties of implementations using the static properties of a specification FSM. In this paper, we will find answers to the following questions on UIO sequences.

(i) Assuming that an implementation could be arbitrarily faulty, what is the measure of goodness of a UIO sequence?

(ii) In the presence of faults, how good are UIO sequences of minimal lengths?

(iii) Can non-minimal UIO sequences with additional properties verify states of a faulty implementation?

(iv) Are two UIO sequences of identical length for the same state equally good?

(v) How to compute the upper bound on the measure of goodness of UIO sequences in a given machine?

The above questions lead to a notion of fault-tolerance capability of UIO sequences. That is, to what extent can a UIO sequence tolerate implementation faults and retain its state verification property? We begin our study with a review of why a UIO sequence fails to verify a state in an implementation. Such a review motivates us to define the concept of strength of a UIO sequence. The strength of a UIO sequence is a quantitative measure of its fault-tolerant capability. 
In Section 2, we state some graph theoretic terms, a basic test sequence, and a fault model. In Section 3, we review how state faults can be masked under various conditions. In Section 4, we present the idea of fault-tolerant UIO sequences and present an algorithm to generate all UIO sequences of maximal strengths. Some concluding remarks are given in Section 5 .

\section{Protocol Specification and Test Sequence}

We organize this section in three parts. In the first part, we present a few FSM and graph-theoretic terms. The general structure of a test sequence is outlined in the second part. Assumptions about an implementation and a fault model are stated in the third part.

\subsection{FSM and Graph-theoretic Terms}

A finite-state machine $M$ is a tuple: $M=(S, I, O, \delta, \lambda)$, where $S=\left\{s_{1}, \ldots, s_{n}\right\}$ is a finite set of states; $I=\left\{a_{1}, \ldots, a_{p}\right\}$ is a finite set of inputs; $O=\left\{b_{1}, \ldots, b_{r}\right\}$ is a finite set of outputs; $\delta: S \times I \rightarrow S$ is the state transition function; and $\lambda: S \times I \rightarrow O$ is the output function.

For convenience we extend the domain of $\lambda$ and $\delta$ from an input symbol to a string of symbols. For instance, for state $s_{i}$ and input sequence $x=a_{1}, \ldots, a_{k}$, the corresponding output sequence is denoted by $\lambda\left(s_{i}, x\right)$ and the final state is denoted by $\delta\left(s_{i}, x\right)$. Also, we extend the transition and output functions from single states to sets of states. That is, if $Q$ is a set of states and $x$ is an input sequence, then $\delta(Q, x)=\{\delta(s, x) \mid s \in Q\}$, and $\lambda(Q, x)=\{\lambda(s, x) \mid s \in Q\}$.

An FSM $M$ is viewed as a directed graph $G=(V, E)$, where the set of vertices $V=\left\{v_{1}, \ldots, v_{n}\right\}$ represents the set $S$ of states of $M$, and a set of edges $E=\left\{\left(v_{j}, v_{k} ; a / b\right) \mid v_{j}, v_{k} \in V\right\}$ represents all transitions in $M$. Each edge $e_{j k}=$ $\left(v_{j}, v_{k} ; a / b\right) \in E$ represents a state transition from $s_{j}$ to $s_{k}$ with input $a$ and output $b$. For the sake of brevity, when $\lambda$ and $\delta$ are known, we also denote a transition by a pair $\left(v_{j}, a\right)$. Notation $x @ y$ denotes the concatenation of strings $x$ and $y$.

We denote an implementation of $M$ by $M^{\prime}=\left(S, I, O^{\prime}, \delta^{\prime}, \lambda^{\prime}\right)$. Though we do not know the next state function $\delta^{\prime}$ and output function $\lambda^{\prime}$ of the implementation, we use them only for explanatory purpose and not to compute any state or output.

\subsection{Basic Test Sequence}

In FSM-based testing, the basic strategy is to apply the following three steps for all states and inputs. First, move the implementation to some state $s_{i}$ and apply an input $a$. Second, verify that the implementation produces the desired output $\lambda\left(s_{i}, a\right)$. Third, verify that the implementation has moved to state $\delta\left(s_{i}, a\right)$ by applying 
a state verification sequence to the implementation and observing the corresponding response.

Let $T X\left(s_{j}, s_{i}\right)$ denote an input sequence that transfers machine $M$ from state $s_{j}$ to $s_{i}$. Let $V S\left(s_{k}\right)$ denote an input sequence that verifies that $M$ is in state $s_{k}$. We leave out the output parts from these sequences, because they can be readily computed from the specification machine. Let $\Omega\left(s_{k}, V S\left(s_{k}\right)\right)$ denote the set of transitions executed by $M$ while $V S\left(s_{k}\right)$ is applied in state $s_{k}$.

Assume that implementation $M^{\prime}$ is in a known state $s_{j}$ and we want to test the output and next state functions associated with transition $\left(s_{i}, a\right)$. Thus, a basic test sequence for state $s_{i}$ and input $a$ is defined as an input sequence:

$B T S\left(s_{i}, a\right)=T X\left(s_{j}, s_{i}\right) @ a @ V S\left(s_{k}\right)$, where $s_{k}=\delta\left(s_{i}, a\right)$.

The three commonly used state verification sequences are distinguishing sequence [KOHA 78], Unique Input/Output (UIO) sequence [SD 88], and pair-wise distinguishing sequence (W-sequence) [CHO 78], [FBK 91]. The shortest path consisting of verified transitions from $s_{j}$ to $s_{i}$ or a reset input is generally selected to be a transfer sequence $T X\left(s_{j}, s_{i}\right)$.

The subsequence $a @ V S\left(s_{k}\right)$ of $B T S\left(s_{i}, a\right)$ is largely known as a test segment. Thus, a complete test sequence for a machine is an appropriate concatenation of a collection of test segments and transfer sequences. For the purpose of minimizing the length of the test sequence, various kinds of optimization techniques involving overlapping of test segments can be used [ADLU 91], [MP 93], [UZ 93].

\subsection{Assumptions and Fault Model}

We assume that a protocol behavior is modeled as a completely specified, deterministic, reduced, and strongly connected finite state machine. We make the following assumptions about an implementation.

(i) An implementation has the same number of states as the specification.

(ii) Faults in an implementation are restricted to output $(\lambda)$ and state $(\delta)$ functions.

(iii) All the faults in an implementation are permanent.

\section{$3 \quad$ Fault Masking}

Assume that an implementation is in state $s_{i}$ and we want to test the output and next state functions for input $a$ in state $s_{i}$. In the UIO method, we need a basic test sequence of the following form:

$\operatorname{BTS}\left(s_{i}, a\right)=a @ I^{\prime}\left(s_{k}\right)$, where $s_{k}=\delta\left(s_{i}, a\right)$ and $I^{\prime} / \lambda\left(s_{k}, I^{\prime}\right)$ is a UIO sequence for state $s_{k}$.

An output fault can be readily detected if the observed output $\lambda^{\prime}\left(s_{i}, a\right)$ is not equal to $\lambda\left(s_{i}, a\right)$. However, detecting a state fault is a non-trivial task in spite of using a 
state verification sequence. This is due to the fact that one fault can mask another [CVI 89], [LS 92], [MP 94]. In the following, we illustrate three kinds of masking:

K1: Output faults in some transitions can mask a state fault in some other transition;

K2: State faults in some transitions can mask a state fault in some other transition.

K3: A state fault masks itself depending on the structure of the FSM.

\section{A. K1 and K2 Type of Fault Masking}

Consider the following scenario. Let the implementation of $\delta\left(s_{i}, a\right)$ be faulty, that is, $\delta\left(s_{i}, a\right)=s_{k}, \delta^{\prime}\left(s_{i}, a\right)=s_{j}$, and $s_{j} \neq s_{k}$. Since we are interested in verifying state $s_{k}$, let $I^{\prime} / \lambda\left(s_{k}, I^{\prime}\right)$ be a UIO sequence for state $s_{k}$. Now we have the following four cases.

Case 1: Output functions of all transitions in $\Omega\left(s_{j}, I^{\prime}\left(s_{j}\right)\right)$ are correctly implemented. Thus, $\lambda^{\prime}\left(s_{j}, I^{\prime}\right)=\lambda\left(s_{j}, I^{\prime}\right)$.

Case 2: Some output functions of transitions in $\Omega\left(s_{j}, I^{\prime}\left(s_{j}\right)\right)$ are faulty such that $\lambda^{\prime}\left(s_{j}, I^{\prime}\right)=\lambda\left(s_{k}, I^{\prime}\right)$.

Case 3: All state transitions in $\Omega\left(s_{j}, I^{\prime}\left(s_{j}\right)\right)$ are correctly implemented.

Case 4: Some state transitions in $\Omega\left(s_{j}, I^{\prime}\left(s_{j}\right)\right)$ are faulty, but the associated outputs are correctly implemented.

In Case $1, \lambda\left(s_{k}, I^{\prime}\right) \neq \lambda^{\prime}\left(s_{j}, I^{\prime}\right)$, because $I^{\prime} / \lambda\left(s_{k}, I^{\prime}\right)$ is a UIO sequence for $s_{k}$. Since the observed sequence $\lambda^{\prime}\left(s_{j}, I^{\prime}\right)$ is different from the expected sequence $\lambda\left(s_{k}, I^{\prime}\right)$, we can deduce that implementation of $\delta\left(s_{i}, a\right)$ is faulty.

However, in Case 2, faults in the implementation of output functions of some transitions in $\Omega\left(s_{j}, I^{\prime}\left(s_{j}\right)\right)$ produce an expected output sequence, thereby effectively masking the state fault $\delta^{\prime}\left(s_{i}, a\right)=s_{j}$. Thus, Case 2 results in a $K 1$ type of fault masking.

The implication of Case 3 is similar to that of Case 1 or Case 2 depending on the correctness of implementation of the output functions.

Case 4 leads to a $K 2$ type of fault masking, because the correct output of the faulty transitions in $\Omega\left(s_{j}, I^{\prime}\left(s_{j}\right)\right)$ produce the expected output, thereby effectively masking the fault under consideration.

Example 1: In Fig. 1, we illustrate the $K 1$ and $K 2$ types of fault masking. Fig. 1(a) shows a part of a specification. We assume that $a b c / x y z_{1}$ and $a b c / x y z_{2}$ are the UIO sequences of states $s 2$ and $s 3$, respectively. Figs. 1(b) and (c) show two kinds of erroneous implementations. We focus on the implementation of the edge $(s 1, s 2 ; d / w)$. In both the implementations, edge $(s 1, s 2 ; d / w)$ has been implemented as $(s 1, s 3 ; d / w)$. We denote the faulty implementation of this transition by fault 
f1. Additionally, edge $(s 7, s 9 ; c / z 2)$ has been implemented as $(s 7, s 9 ; c / z 1)$ with an output fault in Fig. 1(b). This output fault is denoted as $f 2$. Edge $(s 5, s 7 ; b / y)$ has been implemented as $(s 5, s 6 ; b / y)$ with a state fault in Fig. 1(b). This output fault is denoted as $f 3$. It is easy to see that the state fault $f 1$ has been masked by the output fault $f 2$ and state fault $f 3$ in Fig. 1(b) and Fig. 1(c), respectively.

\section{B. K3 Type of Fault}

In the $K 3$ type of fault [CVI 89], a state fault can mask itself depending on the structure of the machine. Let the transition $\left(s_{i}, s_{j} ; a / b\right)$ be implemented as $\left(s_{i}, s_{i} ; a / b\right)$. If there exists an input sequence $I^{\prime}$ such that

(i) $\lambda\left(\delta\left(s_{j}, a\right), I^{\prime}\right)=\lambda\left(s_{i}, I^{\prime}\right)$ and

(ii) $a @ I^{\prime} / \lambda\left(s_{j}, a @ I^{\prime}\right)$ is a UIO sequence for $s_{j}$,

the state fault masks itself. Thus, given an FSM and a UIO sequence for each state, it is possible to check whether $K 3$ types of faults in an implementation could go undetected. The simplest way to avoid the $K 3$ type of fault masking is not to select a UIO sequence satisfying the above properties.

In the following, we discuss an approach to cope with the $K 1$ type of fault masking by introducing the concept of strength of a UIO sequence.

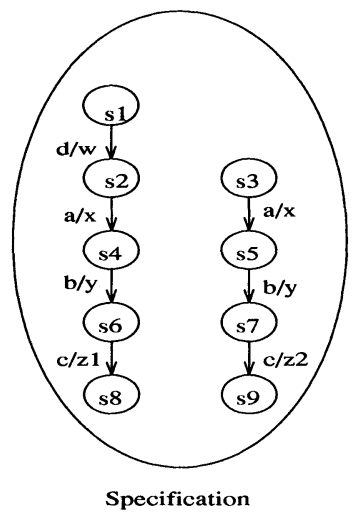

(a)

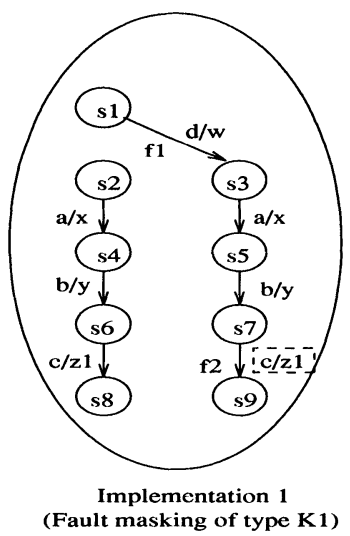

(b)

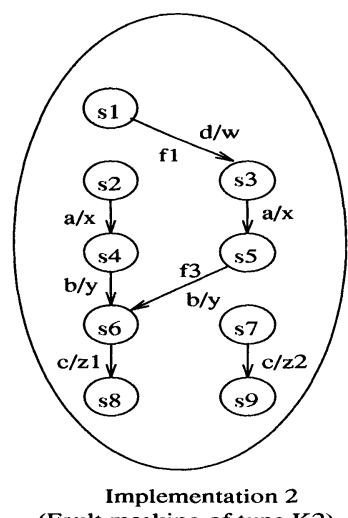

(c)

Figure 1: Illustration of Fault Masking.

\section{Fault-tolerance Capability of a UIO Sequence}

In this section, we define the strength of a UIO sequence and give an algorithm to compute all UIO sequences of maximal strength in an FSM. 


\subsection{Strength of a UIO Sequence}

Definition: Given a state $s$ in a machine $M$ and input sequence $I^{\prime}$, the transition trace $T T\left(s, I^{\prime}\right)$ is defined as the sequence of transitions traversed in $M$ as a result of applying $I^{\prime}$ at $s$.

Definition: Let $s_{i}$ and $s_{j}$ be two states and $I^{\prime}$ be an input sequence in a machine $M$. Let $O_{i}=\lambda\left(s_{i}, I^{\prime}\right)$ and $O_{j}=\lambda\left(s_{j}, I^{\prime}\right)$. The difference between the two output sequences $O_{i}$ and $O_{j}$, denoted by $O_{i} \sim O_{j}$, is defined as a sequence of 0 and 1 , such that the $k$ th. symbol of $O_{i} \sim O_{j}$ is a 1 if and only if:

(i) the $k$ th transitions in $T T\left(s_{i}, I^{\prime}\right)$ and $T T\left(s_{j}, I^{\prime}\right)$ have different outputs and

(ii) in case the $k$ th. transition in $T T\left(s_{i}, I^{\prime}\right)$ is identical to any of its predecessors in $T T\left(s_{i}, I^{\prime}\right)$, its identical predecessor has not contributed a 1 to $O_{i} \sim O_{j}$, and

(iii) in case the $k$ th. transition in $T T\left(s_{j}, I^{\prime}\right)$ is identical to any of its predecessors in $T T\left(s_{j}, I^{\prime}\right)$, its identical predecessor has not contributed a 1 to $O_{i} \sim O_{j}$.

The number of $1 \mathrm{~s}$ in $O_{i} \sim O_{j}$ is called the degree of difference between the two output sequences and is denoted by $D o D\left(O_{i}, O_{j}\right)$.

Remark: In the above definition, the first condition for assigning a 1 to the $k$ th. entry of the difference sequence is obvious. The last two conditions allow us to consider the output fault of a transition exactly once, even though the transition may appear many times in the transition trace.

Definition: Let $M=(S, I, O, \delta, \lambda)$ be an FSM. The strength of a UIO sequence $I^{\prime} / \lambda\left(s_{i}, I^{\prime}\right)$ for state $s_{i}$ is the minimum of $D o D\left(\lambda\left(s_{i}, I^{\prime}\right), \lambda\left(s_{j}, I^{\prime}\right)\right), \forall s_{j} \in S$ and $s_{j} \neq s_{i}$. We denote the strength of a UIO sequence $U I O_{k}$ for state $s_{i}$ by $\operatorname{strength}\left(s_{i}, U I O_{k}\right)$.

Remark: Obviously, the minimum strength of any UIO sequence for any state is one. In case a state has many UIO sequences, their strengths could be different.

The motivation for defining the strength of a UIO sequence is as follows. Consider transition $\left(s_{i}, s_{j} ; a / b\right)$ which has been incorrectly implemented as $\left(s_{i}, s_{k} ; a / b\right)$ with a state fault. Assume that $U I O_{s_{j}}=I^{\prime} / \lambda\left(s_{j}, I^{\prime}\right)$ is used to verify state $s_{j}$. If $\operatorname{strength}\left(s_{j}, U I O_{s_{j}}\right)=1$, one output fault in the set of transitions $\Omega\left(s_{k}, I^{\prime}\left(s_{k}\right)\right)$ is necessary to mask the state fault. If $\operatorname{strength}\left(s_{j}, U I O_{s_{j}}\right)=2$, two output faults in the set of transitions $\Omega\left(s_{k}, I^{\prime}\left(s_{k}\right)\right)$ are necessary to mask the state fault. In general, if strength $\left(s_{j}, U I O_{s_{j}}\right)=m, m \geq 1, m-1$ output faults in $\Omega\left(s_{k}, I^{\prime}\left(s_{k}\right)\right)$ can mask the state fault. Thus, higher the strength of a UIO sequence, more output faults are necessary to mask a state fault. Theoretically, in order to detect the state fault, we need a UIO sequence whose strength is more than the number of output faults in an implementation.

Remark: All minimal length UIO sequences have a strength of one. Thus, a single output fault can render them ineffective.

Example 2: Consider machine $M 1$ and its implementation in Figs. 2(a) and (b), respectively. Transition $\left(s_{6}, s_{3} ; b / 1\right)$ has been incorrectly implemented with a 
state fault as $\left(s_{6}, s_{5} ; b / 1\right)$ and transition $\left(s_{1}, s_{3} ; a / 1\right)$ has been incorrectly implemented with an output fault as $\left(s_{1}, s_{3} ; a / 0\right)$. We want to test the implementation of state transition $\left(s_{6}, s_{3} ; b / 1\right)$. We will consider two cases, such that the first case involves a UIO sequence with a strength of one and the second case involves a UIO sequence with a strength of two. In both the cases, we assume that the implementation is in state $s_{6}$.

Case 1: Consider the minimal length UIO sequence $a a a / 000$ for state $s_{3}$.

$\lambda\left(s_{1}, a a a\right)=100, \lambda\left(s_{2}, a a a\right)=010$,

$\lambda\left(s_{3}, a a a\right)=000, \lambda\left(s_{4}, a a a\right)=110$,

$\lambda\left(s_{5}, a a a\right)=001, \lambda\left(s_{6}, a a a\right)=101$.

$\operatorname{DoD}\left(\lambda\left(s_{3}, a a a\right) \sim \lambda\left(s_{1}, a a a\right)\right)=1$,

$\operatorname{DoD}\left(\lambda\left(s_{3}, a a a\right) \sim \lambda\left(s_{2}, a a a\right)\right)=2$,

$\operatorname{DoD}\left(\lambda\left(s_{3}, a a a\right) \sim \lambda\left(s_{4}, a a a\right)\right)=2$,

$\operatorname{DoD}\left(\lambda\left(s_{3}, a a a\right) \sim \lambda\left(s_{5}, a a a\right)\right)=1$,

$\operatorname{DoD}\left(\lambda\left(s_{3}, a a a\right) \sim \lambda\left(s_{6}, a a a\right)\right)=2$.

Therefore, strength $\left(s_{3}, a a a / 000\right)=1$. A basic test sequence to test the implementation of $\left(s_{6}, s_{3} ; b / 1\right)$ is:

$\operatorname{BTS}\left(s_{3}, b\right)=b @ a a a$,

where input $b$ is expected to take the implementation to state $s_{3}$ and the $a a a$ sequence is expected to verify that the implementation indeed moves to state $s_{3}$. However, input $b$ takes the implementation to state $s_{5}$ and the sequence $a a a$ in state $s_{5}$ produces the "expected" output 000 , because transition $\left(s_{1}, s_{3} ; a / 1\right)$ has been implemented as $\left(s_{1}, s_{3} ; a / 0\right)$ with an output fault. Thus, the basic test sequence fails to detect the state fault due to a $K 1$ type of fault masking.

Case 2: Consider a non-minimal length UIO sequence aaaba/00010 for state $s_{3}$. From $M 1$,

$\lambda\left(s_{1}, a a a b a\right)=10001, \lambda\left(s_{2}, a a a b a\right)=01001$,

$\lambda\left(s_{3}, a a a b a\right)=00010, \lambda\left(s_{4}, a a a b a\right)=11010$,

$\lambda\left(s_{5}, a a a b a\right)=00111, \lambda\left(s_{6}, a a a b a\right)=10111$.

$\operatorname{DoD}\left(\lambda\left(s_{3}, a a a b a\right) \sim \lambda\left(s_{1}, a a a b a\right)\right)=3$,

$\operatorname{DoD}\left(\lambda\left(s_{3}, a a a b a\right) \sim \lambda\left(s_{2}, a a a b a\right)\right)=3$,

$\operatorname{DoD}\left(\lambda\left(s_{3}, a a a b a\right) \sim \lambda\left(s_{4}, a a a b a\right)\right)=2$,

$\operatorname{DoD}\left(\lambda\left(s_{3}, a a a b a\right) \sim \lambda\left(s_{5}, a a a b a\right)\right)=2$,

$D o D\left(\lambda\left(s_{3}, a a a b a\right) \sim \lambda\left(s_{6}, a a a b a\right)\right)=3$.

Therefore, strength $\left(s_{3}, a a a b a / 00010\right)=2$. A basic test sequence to test the implementation of $\left(s_{6}, s_{3} ; b / 1\right)$ is:

$\operatorname{BTS}\left(s_{3}, b\right)=b @ a a a b a$,

where the first input $b$ is expected to take the implementation to state $s_{3}$ and the $a a a b a$ sequence is expected to verify that the implementation indeed moves to state $s_{3}$. However, input $b$ takes the implementation to state $s_{5}$ and the sequence aaaba in 
state $s_{5}$ produces the output 00011 . Since the output of the implementation 00011 differs from the expected output $\lambda\left(s_{3}, a a a b a\right)=00010$, one can conclude that there is a state fault in the implementation of transition $\left(s_{6}, s_{3} ; b / 1\right)$. That is, the basic test sequence using a UIO sequence of strength two can tolerate one output fault in the implementation of any transition.

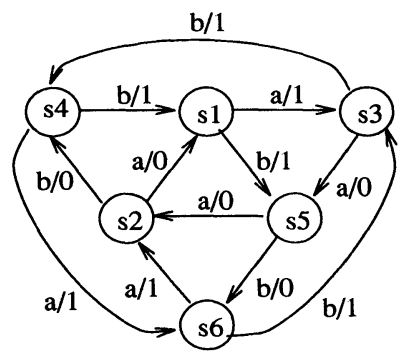

(a) Specification Machine M1

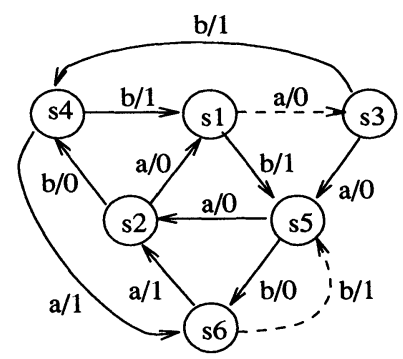

(b) Implementation of M1

Figure 2: A specification [UZ 93] and a faulty implementation.

\subsection{Generation of UIO Sequences of Maximal Strength}

One can obtain UIO sequences of minimal lengths using the algorithm in [SD 88]. Given a UIO sequence for a state, one can always obtain a UIO sequence of longer length for the same state and check its strength. In order to generate UIO sequence of higher strength, the UIO sequence needs to be made longer. However, it is difficult to define a termination condition for this approach.

Therefore, we will take a different approach to obtaining UIO sequences of higher strength. We will modify another algorithm to compute UIO sequences [NAIK 94] for this purpose. We first introduce a path vector and perturbation of a path vector. Based on the perturbation idea, next we define a UIO-tree. We incorporate three termination conditions into the perturbation process so that the algorithm terminates after generating all UIO sequences of maximal strengths.

Definition: Given an FSM $M$, a path vector (PV) is a collection of state pairs, $\left(v_{1} / v_{1}^{\prime}, \ldots, v_{i} / v_{i}^{\prime}, \ldots, v_{k} / v_{k}^{\prime}\right)$, with the following properties:

(1) $v_{i}$ and $v_{i}^{\prime}$ denote the head and tail state, respectively, of a path, where a path is a sequence of state transitions and

(2) an identical sequence of input/output is associated with all the paths in the path vector.

Given a path vector $P V=\left(v_{1} / v_{1}^{\prime}, \ldots, v_{i} / v_{i}^{\prime}, \ldots, v_{k} / v_{k}^{\prime}\right)$, the initial vector $(I V)$ is the collection of head states of $P V$, that is $I V(P V)=\left(v_{1}, \ldots, v_{i}, \ldots, v_{k}\right)$. Similarly, the current vector $(C V)$ is the collection of tail states of $P V$, that is $C V(P V)=$ $\left(v_{1}^{\prime}, \ldots, v_{i}^{\prime}, \ldots, v_{k}^{\prime}\right)$. 
A path vector is said to be a singleton vector if it contains exactly one state pair. A path vector $P V=\left(v_{1} / v_{1}^{\prime}, \ldots, v_{i} / v_{i}^{\prime}, \ldots, v_{k} / v_{k}^{\prime}\right)$ is said be a homogeneous vector if all members of $C V(P V)$ are identical. It may be noted that a singleton vector is also a homogeneous vector.

For an $n$-state machine, we define a unique initial path vector: $\left(s_{1} / s_{1}, \ldots, s_{i} / s_{i}\right.$, $\left.\ldots, s_{n} / s_{n}\right)$ such that a null path is associated with all state pairs.

Now we introduce the idea of vector perturbation. That is, given a path vector $P V$ and an edge label $a / b$, how to compute a new vector $P V^{\prime}$ from $P V$ and $a / b$.

Definition: Given a path vector $P V=\left(v_{1} / v_{1}^{\prime}, \ldots, v_{i} / v_{i}^{\prime}, \ldots, v_{k} / v_{k}^{\prime}\right)$ and an edge label $a / b$, perturbation of $P V$ with respect to edge label $a / b$, denoted by $P V^{\prime}=$ $\operatorname{pert}(P V, a / b)$ is defined as:

$P V^{\prime}=\left\{v_{i} / v_{i}^{\prime \prime} \mid v_{i}^{\prime \prime}=\delta\left(v_{i}^{\prime}, a\right) \wedge \lambda\left(v_{i}^{\prime}, a\right)=b \wedge v_{i} / v_{i}^{\prime} \in P V\right\}$.

Given a reduced machine and its initial path vector, we can infinitely perturb all the path vectors for all edge labels. One can imagine the perturbation function $P V^{\prime}=\operatorname{pert}(P V, a / b)$ as an arc from a node $P V$ to a new node $P V^{\prime}$ with edge label $a / b$. Also, given a $P V$ and a set of edge labels $L$, we can arrange the new $|L|$ nodes $\operatorname{pert}(P V, a / b), \forall a / b \in L$ on one level. That is, all the path vectors of a given machine can be arranged in the form of a tree with successive levels $1,2, \ldots \ldots \infty$. Such a tree is called a UIO-tree.

Note: In the graphical representation of a path vector $P V=\left(v_{1} / v_{1}^{\prime}, \ldots, v_{i} / v_{i}^{\prime}, \ldots\right.$, $\left.v_{k} / v_{k}^{\prime}\right)$ in a UIO-tree, we represent $P V$ in two rows of states. The top row denotes $I V(P V)$ and the bottom row denotes $C V(P V)$.

Theoretically, a UIO-tree is a tree with infinite levels. However, we need to prune the tree based on some conditions, called pruning conditions. After each perturbation $P V^{\prime}=\operatorname{pert}(P V, a / b)$, we check the following pruning conditions:

C1: $C V\left(P V^{\prime}\right)$ is a homogeneous vector containing more than one element.

C2: On the path from the initial node to $P V$, there exists $P V^{\prime \prime}$ such that $P V^{\prime} \subseteq P V^{\prime \prime}$.

C3: On the path from the initial node to $P V$, there exists a singleton node and a node $P V^{\prime \prime}$ such that $P V^{\prime} \subseteq P V^{\prime \prime}$. (Note: the singleton node and $P V^{\prime \prime}$ can be the same node.)

If one of the pruning conditions is satisfied, we declare $P V^{\prime}$ to be a terminal node. Now we explain why $C 1, C 2$ and $C 3$ are the required termination conditions. Let us consider condition $C 1$. If $C V\left(P V^{\prime}\right)$ is a homogeneous vector containing more than one element, any further perturbation of the path from the initial node to $P V^{\prime}$ will not lead to any UIO sequence. Condition $C 2$ states that there is no need of perturbing a node if the node or a superset of the node appears before. This is because, if the node or its superset appears before, then it has already been perturbed in all possible ways and perturbing it once again will not lead to new information. Condition $C 3$ deals with UIO sequences. If a UIO-tree contains a singleton node, then the sequence of input/output associated with the path from the initial node to the singleton node 
is a UIO sequence for the head state of the node. If we are simply interested in UIO sequences of minimal lengths, we can stop perturbing a singleton node [NAIK 94]. However, we are interested in UIO sequences of maximal strengths. Therefore, we further perturb a singleton node until we know that further perturbation will not add extra information to the UIO sequence. This happens when the singleton node appears before either as itself or as a subset of a larger node. In the rest of the paper, we use the terms path vector and node in a UIO-tree interchangeably. In the following, we present an algorithm to compute maximal strength UIO sequences.

Algorithm 1: Generating UIO Sequences of Maximal Strength.

Input: $M=(S, I, O, \delta, \lambda)$ and $L$.

Output: UIO sequences of maximal strengths.

Method: Execute the following steps.

Step 1: Let $\Psi$ be the set of nodes denoting path vectors in the UIO-tree. Initially, $\Psi$ contains the initial vector marked as non-terminal.

Step 2: Find a non-terminal member $\psi \in \Psi$ which has not been perturbed. If no such member exists, then go to Step $\mathbf{5}$.

Step 3: Compute $\psi^{\prime}=\operatorname{pert}\left(\psi, a_{i} / b_{i}\right)$ and add $\psi^{\prime}$ to $\Psi, \forall a_{i} / b_{i} \in L$. Mark $\psi$ to be perturbed. Update the UIO-tree.

Step 4: If $\operatorname{pert}\left(\psi, a_{i} / b_{i}\right)$, computed in Step 3, satisfies condition $C 1, C 2$, or $C 3$, then mark $\operatorname{pert}\left(\psi, a_{i} / b_{i}\right)$ as a terminal node and go to Step 2.

Step 5: Collect all the UIO sequences for every state and compute their strengths.

Step 6: If a state has at least one UIO sequence, select a UIO sequence of maximal strength. After selecting UIO sequences of maximal strength, terminate the algorithm. End of Algorithm 1

Theorem: Given a machine $G=(V, E)$, a state $v$ has a UIO sequence iff the UIO-tree has a singleton node $\psi$ such that $v=I V(\psi)$.

A proof of the above theorem can be found in [NAIK 94]. Obviously, the algorithm has an exponential complexity. Termination of the algorithm is guaranteed by the three conditions $C 1, C 2$, and $C 3$.

Example 3: We applied Algorithm 1 to the specification machine of Fig. 2 to compute the UIO-tree. A part of the UIO-tree is shown in Fig. 3. Node $s_{3} / s_{1}$ is a singleton node indicating that the sequence of input/output aaa/000 leading from the initial node to $s_{3} / s_{1}$ is a UIO sequence of minimal length for state $s_{3}$. However, to obtain UIO sequences of higher strength, we further perturb node $s_{3} / s_{1}$ as shown in Fig. 3 to obtain nodes $s_{3} / s_{3}$ and $s_{3} / s_{5}$. Node $s_{3} / s_{3}$ is a terminal node because condition $C 3$ is satisfied. Node $s_{3} / s_{5}$ is further perturbed to obtain nodes $s_{3} / s_{2}$ and $s_{3} / s_{6}$. Node $s_{3} / s_{2}$ is a terminal node because condition $C 3$ is satisfied. We continue perturbing node $s_{3} / s_{6}$ to obtain nodes $s_{3} / s_{2}$ and node $s_{3} / s_{3}$, which are both terminal nodes. For machine $M 1$, the maximal strength of any UIO sequence for any state is two. 


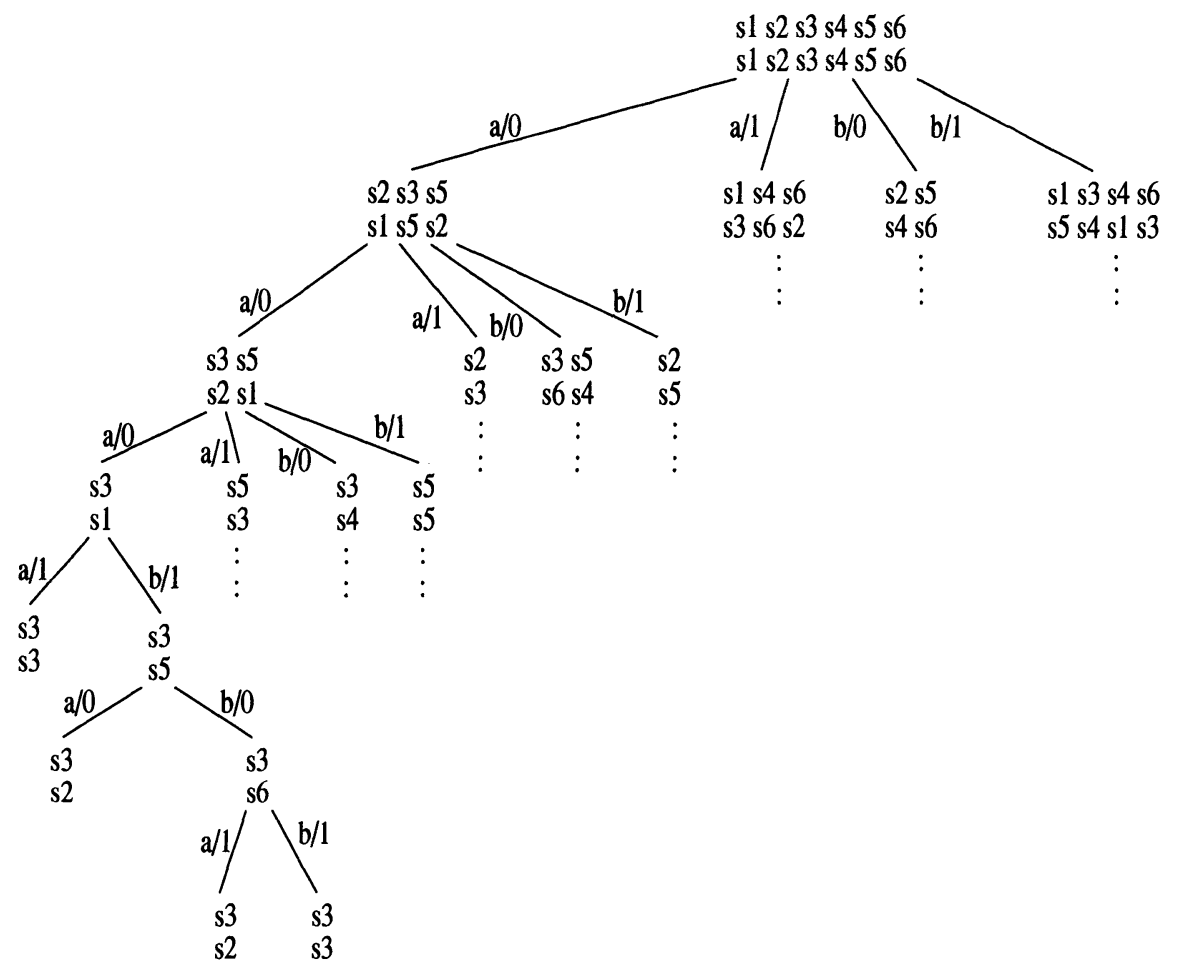

Figure 3: A Partial UIO-tree for specification machine M1. 


\section{Concluding Remarks}

In this paper, we discussed how output and state faults can mask some other state faults by rendering the state verification power of a UIO sequence ineffective. The idea of strength of a UIO sequence was useful in defining the fault-tolerance capability of UIO sequences. UIO sequences of higher strengths are generally longer than the minimal length sequences. Since a minimal length UIO sequence has a strength of one, a single output fault can easily render the UIO sequence useless! Since fault detection, rather than short execution time, is presumed to be the main objective of testing, using the minimal length UIO sequences does not seem to be a good idea. UIO sequences of maximal strengths, though longer than the minimal length UIO sequences, are better than the minimal length UIO sequences.

We believe that our work will lead to new research on test generation involving minimization of test sequences with maximal fault detection for a given FSM specification. Presently, the idea of overlapping of test segments is widely used in minimizing a test sequence. However, our work reveals the requirement for a new way of scheduling test segments.

As an extension of our work, one can apply the fault-tolerance idea to other state verification techniques. In our opinion, this work will provide a quantitative and formal basis for comparing the fault detection capabilities of test sequences generated using the D-, W-, and U-methods. More research is required to be done to generate UIO sequences that can tolerate both output and state faults. 


\section{References}

[KOHA 78] Z. Kohavi, Switching and Finite Automata Theory, Second Ed. New York: McGraw-Hill, 1978.

[CHO 78] T.S. Chow, "Test Design Modeled by Finite State Machine," IEEE Trans. on Software Eng., Vol. SE-4, No. 3, 1978, pp. 178-187.

[SD 88] K. K. Sabnani and A. T. Dahbura, "A Protocol Test Generation Procedure," Computer Networks and ISDN Systems, Vol. 15, No. 4, 1988, pp. 285-297.

[CVI 89] W. Y. L. Chan, S. T. Vuong, and M. R. Ito, "An Improved Protocol Test Generation Procedure Based on UIO's," in Proc. of SIGCOMM'89, 1989, pp. 283-294.

[SL 89 ] D. P. Sidhu and T. K. Leung, "Formal Methods in Protocol Testing: A Detailed Study," IEEE Trans. on Software Eng., Vol. 15, No. 4, April 1989, pp. 413-426.

[ADLU 91] A. V. Aho, A. T. Dahbura, D. Lee, and M. U. Uyar, "An Optimization Technique for Protocol Conformance Test Generation Based on UIO Sequences and Rural Chinese Postman Tours," IEEE Trans. on Communications, Vol. 39, No. 11, Nov. 1991, pp. 1604-1615.

[FBK 91] S. Fujiwara, G. v. Bochmann, F. Khendek, et. al., "Test Selection Based on Finite State Models", IEEE Trans. on Software Eng., SE-17, No. 6, June 1991, pp. 591-603.

[LS 92] F. Lombardi and Y.- N. Shen, "Evaluation and Improvement of Fault Coverage of Conformance Testing by UIO Sequences," IEEE Trans. on Comm., Vol. 40, No. 8, August 1992, pp. 1288-1293.

[UZ 93] H. Ural and K. Zhu, "Optimal Length Test Sequence Generation Using Distinguishing Sequences," IEEE/ACM Trans. on Networking, Vol. 3, No. 1, June. 1993, pp. 358-371.

[MP 93] R. E. Miller and S. Paul, "On the Generation of Minimal-Length Conformance Tests for Communication Protocols," IEEE/ACM Trans. on Networking, Vol. 1, No. 1, Feb. 1993, pp. 116-129.

[MP 94] R. E. Miller and S. Paul, "Structural Analysis of Protocol Specifications and Generation of Maximal Fault Coverage Conformance Test Sequences," IEEE/ACM Trans. on Networking, Vol. 2, No. 5, Oct. 1994, pp. 457-470.

[NAIK 94] K. Naik, "Efficient Computation of Unique Input/Output Sequences in Finite State Machines," submitted for publication. 